\title{
The Control Simulation of the Enterprise on the Basis Metasystem Approach
}

\author{
Alexandr Pishchukhin*, Tatiana Pishchukhina \\ Orenburg State University \\ *Corresponding Author: fit@unpk.osu.ru
}

Copyright $(02013$ Horizon Research publishing all rights reserved.

\begin{abstract}
Enterprise model, as integration of grocery technologies, is represented metasystem. Such point of view allows to put and discus six problems of metasystem approach and to reveal probabilistic model of functioning enterprise based on the queuing system with a flow of demands from the market. Management in such model provides functioning of grocery technologies at a certain level of probability. By means of computer on two-grocery model of the enterprise is proved that such management the accuracy of technology switching - according to market requirements increases. Research showed that the described approach allows to optimize management of the enterprise on a scientific basis, maximizing "metasystem effect".
\end{abstract}

UDC Number: 007:681.518.2

Keywords Metasystem, Tasks of Metasystem Approach, Queuing System, Enterprise Management

\section{Introduction}

Management of manufacturing enterprise - difficult economic process (Pishchukhin, Pogorelov 2007), which demands system modeling and the analysis.

The enterprise at the top level of representation can be viewed as a set of jointly or separately functioning technologies. Because each technology is a complete system for modeling the enterprise needs to consider systems integration into a whole. The theory of such aggregation can be built on the ideology of the metasystem approach, while the principles of operation can be borrowed from queuing systems and used at the bottom level. In this paper we attempt by metasystem approach the upper control level align market demand with the production program of the enterprise. Due to the standard approach on the lower level of sustainability issues are not considered

\section{Theory}

In accordance with systemology J. Klir there are two ways of integration systems (Klir 1985) - on the basis of creation: a structured system or metasystem. In the first case, the system is divided into subsystems, which in turn split into subsystems second level and third level, and so on there between organized relationship. In the second case metasystem is formed on the basis of the substitution rule, when from a set of systems in each time selects one or a group of operating systems.

Structured system characterized by the following properties (Mironov, Pishchukhin 2004): 1)between the elements there is a strong and stable relationship; 2)at a given time is operating all elements of the system; 3 ) the number of elements in the system is determined by the principle of completeness and up-sufficiency;

Differences structured system from metasystem illustrated in Figure 1. These two systems react differently to external stimuli (Pishchukhin, Skamyin 2005): a structured system only change the signals in the internal links, and metasystem, in addition, can replace a functioning system by the rule change, or in the case of malfunction, so the metasystem more tenacious.

Metasystem approach (Pishchukhin et al. 2004)is characterized by three essential features compared with signs stated above and distinguishing it from the traditional system: 1)metasystem elements in big degree are self-sufficient and independent from each other; 2) in metasystem not all elements, and only one, or some group of the chosen at any moment function; 3) in metasystem portfolio systems satisfies absolutely to other criteria and has to be optimum according to them. Therefore that in a practical task to prove legitimacy of metasystem approach it is necessary to find these distinctive signs first of all.

At metasystem approach it is necessary to solve the following standard tasks, not arising at system approach: identification of subranges of effective functioning of systems; assessment and increase of necessary level of readiness of systems to use; development strategy of switching separate or groups of at the same time functioning systems; identification and ensuring the compatibility, the coordinated interaction of systems; optimum redistribution of limited all-system resources; optimum synthesis of metasystem. 
Structured system

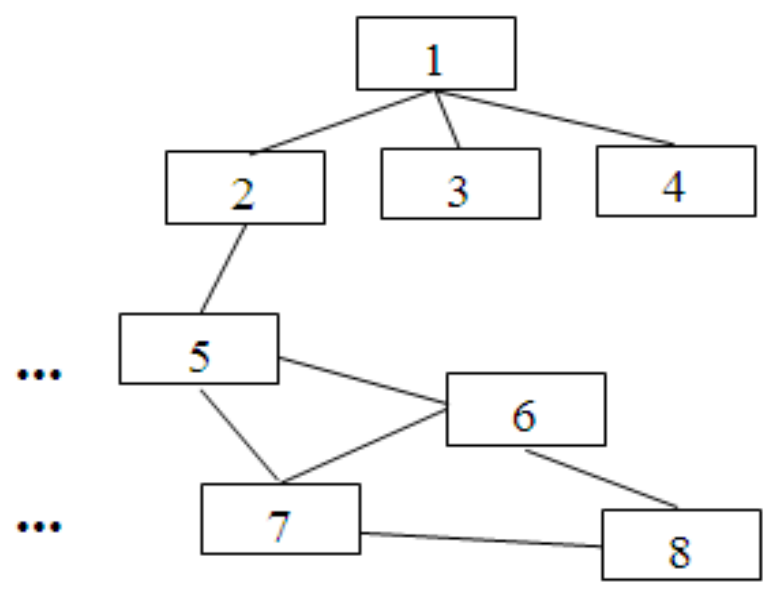

Metasystem

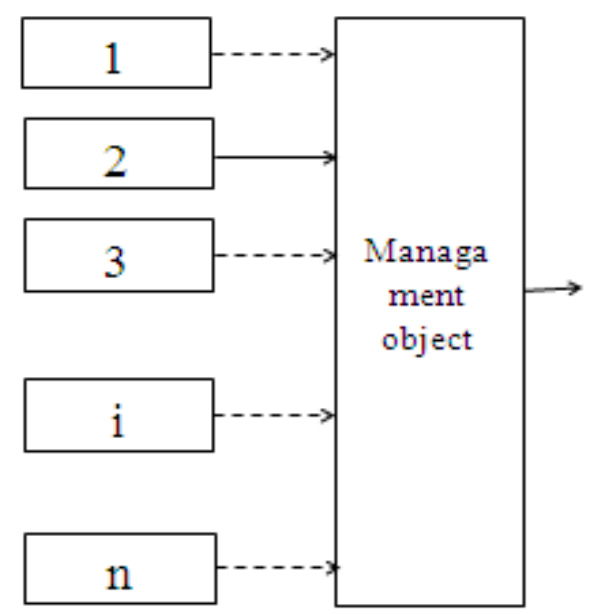

Figure 1. Distinctions between two ways of systems integration

It is most convenient to begin the metasystem analysis with identification subranges of optimum performance of systems as on their basis further it is convenient to develop strategy choice of systems. At the solution of this task it is important to remember that the metasystem arises there and then where and when the range of a solved task is so great that it isn't blocked by use of one system, or this system functions inefficiently in some parts of the general range. Therefore there is a problem of identification of the borders dividing subranges of effective functioning of systems. These subranges can be revealed by means of multidimensional space of signs.

For the enterprise presented as set of technologies, can be the specified signs: the price for products, made on concrete technology, prime cost of this production, demand for this type of production, volume of its release and others. In this space surfaces at which crossing by the representing point reflecting a conditions of the market and the enterprise, it is necessary to switch technology are under construction.

The second task is connected with that there is a period in which not used systems stand idle and, so, lose readiness for use. For the technologies included in a portfolio of the enterprise, loss of readiness is connected with malfunction of the idle equipment, lack of preparations, raw materials, the materials used at this technology, unavailability, for one reason or another, shots.

Readiness can be estimated probability of that the system will function by the time of switching on it. It is possible to operate this probability only creating favorable conditions for emergence of a desirable event, or eliminating the reasons of its not emergence (Pishchukhin et al. 2010).

The third task of metasystem approach is predictive. If somehow it is possible to develop strategy of switching of systems, the moments of the beginning of functioning of each system will be known. It will be possible to prepare them for these moments, optimum spending resources as maintenance of constant readiness of all systems demands unfairly many expenses.

It is possible to project the called strategy in the multidimensional space mentioned in the first task on the basis of making of a predictive trajectory of movement of the point representing a conditions of the market and the enterprise (Pishchukhin et al., 2003).

The fourth task provides some interrelation of systems, for example at structural level when combinations of some systems when functioning give bigger effect, than other their combinations. For example, functioning of manufacturing techniques of any product on the market and the technology using waste arising thus, or functioning of two close technologies with sharply changing demand for each type of production made with their help - "emissions" of the market it is possible to smooth skillfully, flexibly operating release volumes.

The fifth task, arises only at simultaneous functioning of several systems, thus to the forefront there is a need for their coordination, optimization of the resources directed on each system, with the purpose to achieve the greatest efficiency of metasystem. Thus all-system resources go in a bigger measure to that system which most of all increases "metasystem effect".

At last, the sixth task is connected with optimization of a set of systems in metasystem. As criterion it is possible to choose coefficient of specific effect, that is the effect relation from inclusion of this technology in a metasystem portfolio to the expenses connected with its acquisition and development and then the portfolio joins first of all systems with the biggest coefficient of specific effect.

Metasystems can be divided into two big classes (Pishchukhin, Shalkin 2001): consecutive and parallel action. In the first case one functions only - the chosen system, in the second - the chosen group of systems.(Pishchukhin, Korshunova 2009) 


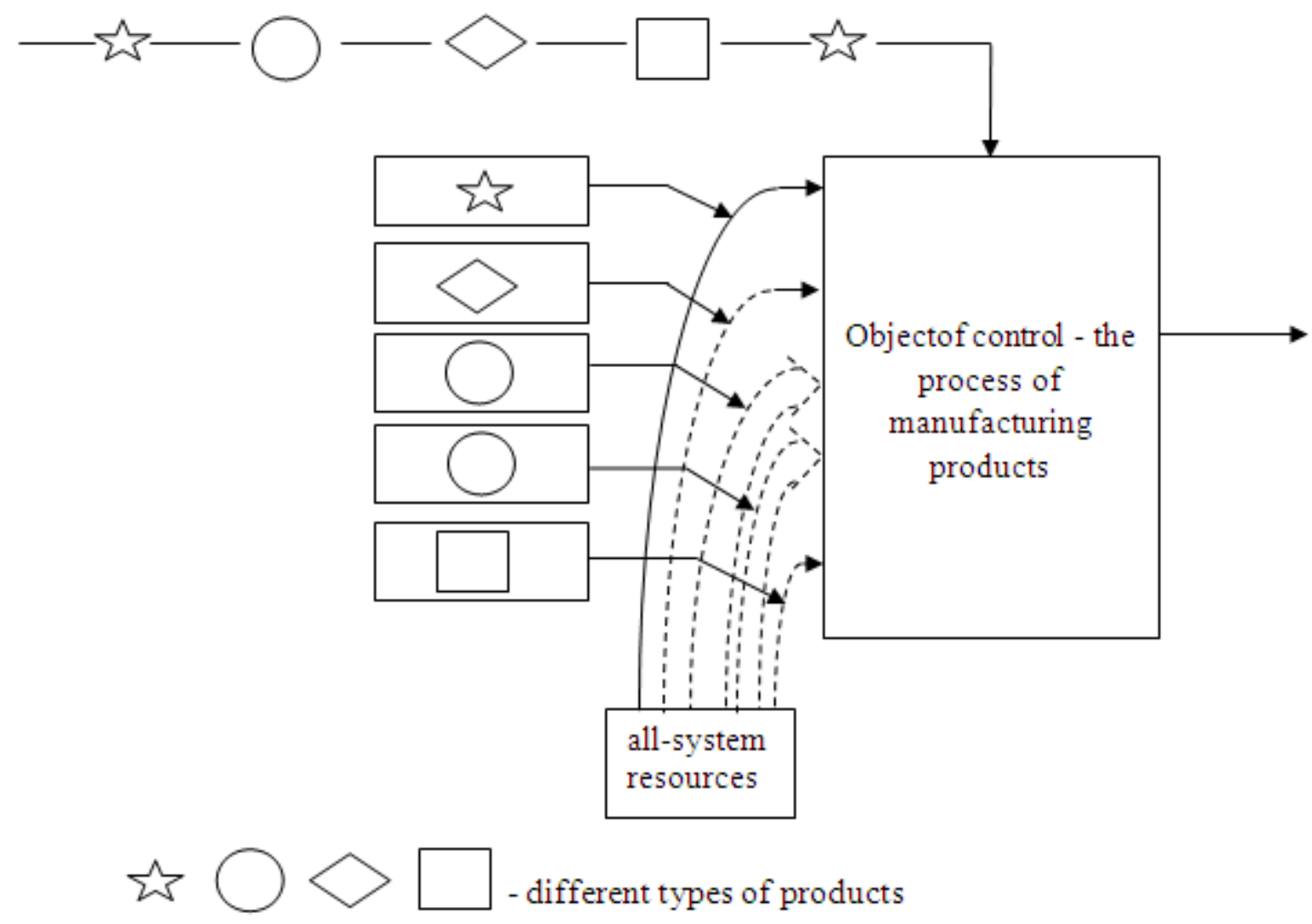

Figure 2. queuing system model of enterprise

\section{Method}

The model of the enterprise is represented in the form of the system of mass service working on a flow of demands for production of this or that type of production, arriving from the market and caused by demand - figure 2 (Pishchukhin, Skamyin 2007).

Figure currently operates top technology and other idle marked dotted line, the third and fourth technology should work in parallel and therefore require an optimum reallocation of resources between them.

\section{The Data and Simulation Results}

The proposed operation of the business model should be the need for rapid adjustment inclusion probabilities for all of the technologies included in the portfolio of the enterprise with the corresponding probability of changes in market demand for the relevant product type. Based on the controllability of the enterprise, you can enter the main feedback that will monitor the actual switching frequency of each structure, memorize it and call with the desired probability of the market. Moreover, this relationship should operate only in the cases of greatest uncertainty, that is, the boundaries between the classes of management situations. An experimental study focused on evaluating the accuracy of inclusions technologies that will boost said feedback.

The pilot scheme was a two consecutively switched technology depicted in Figure 3. Here - setting the frequency switching regulators, $<\mathrm{W}>$ - assessment of probability inclusion of technology. After loop circuit compared with the operation of the circuit if any noise in an external process. Changes in demand without the noise component depicted in Figure 4, and represent two different sine wave with twice the period.

Feedback will only work within a certain range, when the difference between the values of noisy sine less than some $\varepsilon$. Correction circuit with feedback is only by increasing the switching frequency. Errors are calculated as the ratio of modulus of the difference frequency switching noise in the circuits with the switching frequency in the circuit to the last without the noise and frequency were taken as a percentage. The resulting dependence of the experimental error of inclusions is shown in Figure 5. 


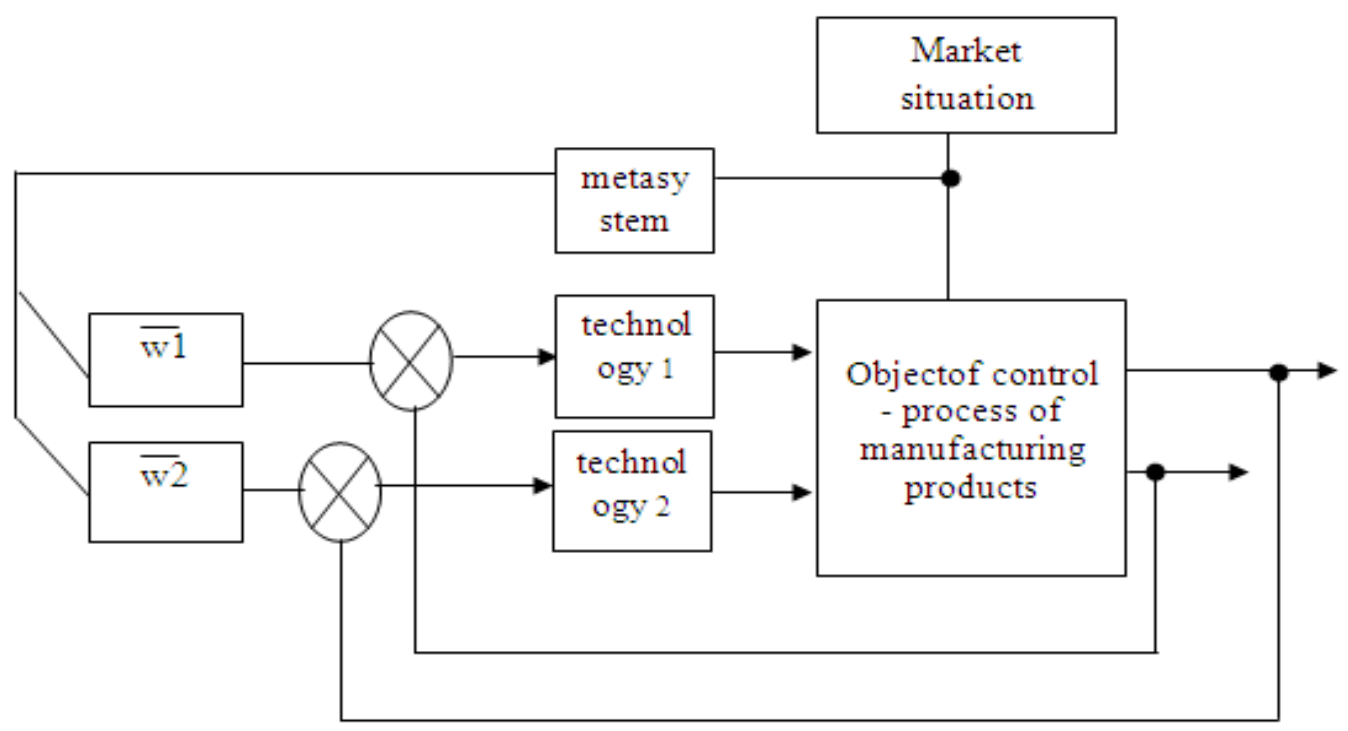

Figure 3. The scheme of two consistently switched technologies

Amplitude

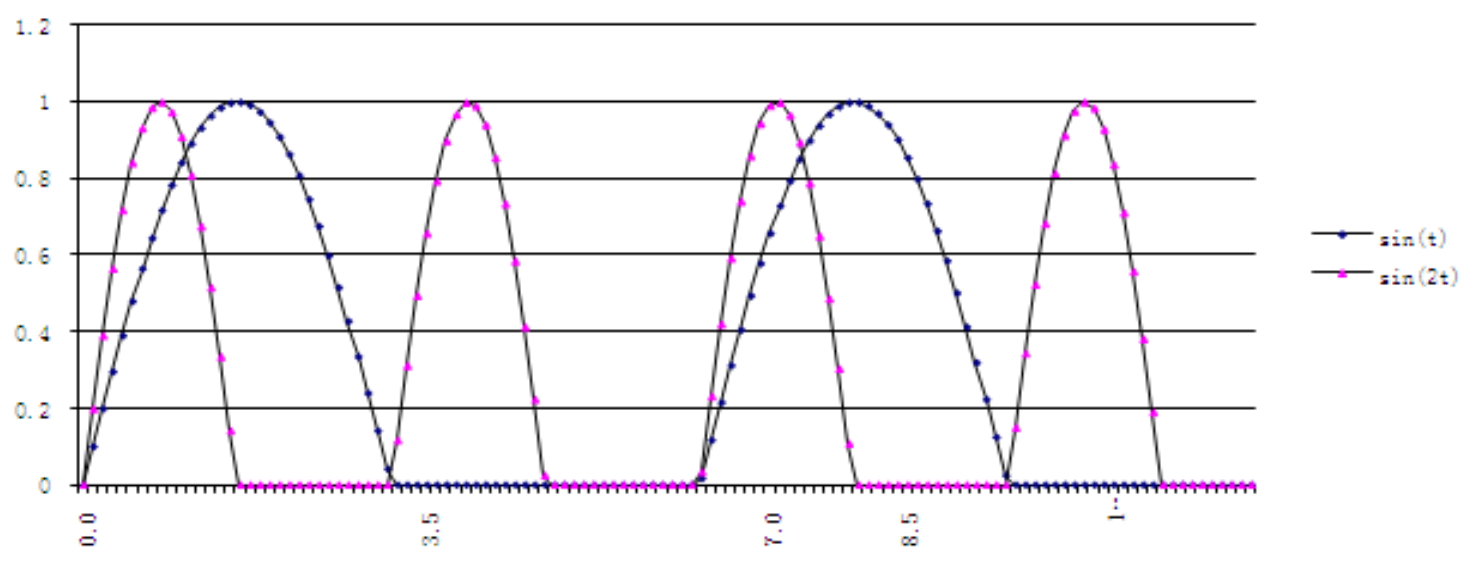

Time

Figure 4. The schedule of change in time of demand for made production

Mistake on inclusions, $\%$

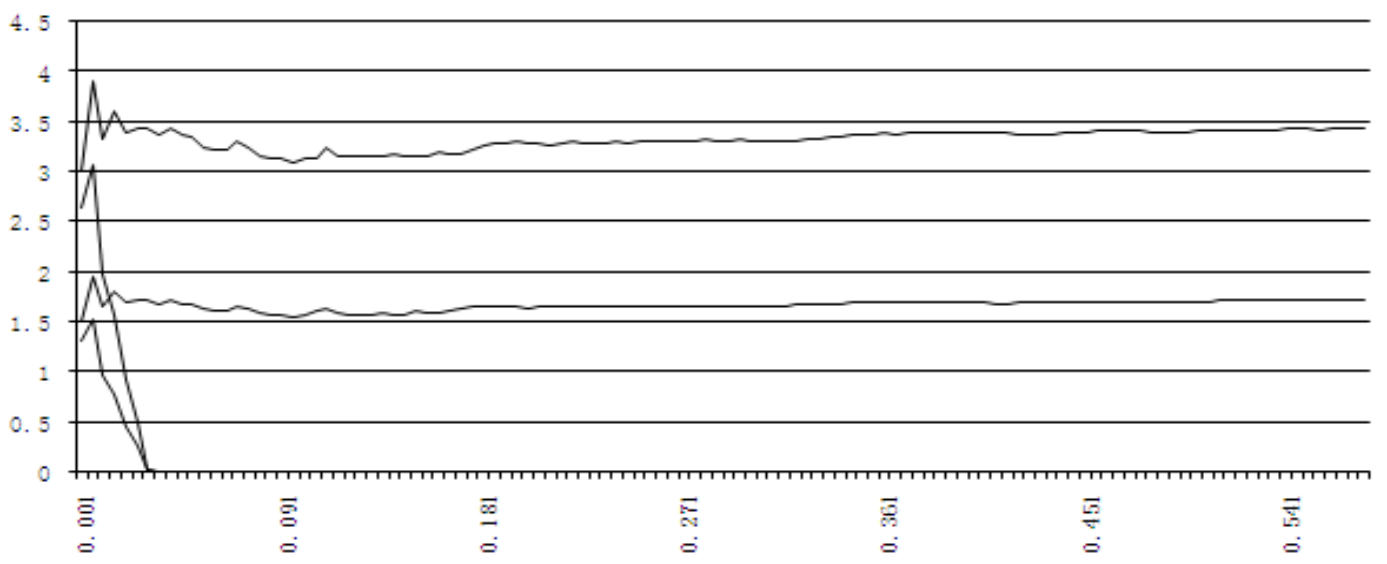

Range of operation the feedbacks $(\varepsilon)$

Figure 5. Dependences of a mistake on the frequency of inclusion technologies 


\section{Discussion}

Apparently from the schedule (figure 5), some mistake at the scheme with feedback takes place only at small, comparable with a maximum level of noise. With growth the mistake quickly decreases and addresses in zero for both included technologies. At the same time in the second scheme of an error of inclusion of technologies differ. Their insignificant dependence on is caused only by noise.

\section{Conclusions}

Thus, metasystem approach is the necessary tool for modeling functioning process of manufacturing enterprise. Management in such model provides maintenance at a certain level of probability functioning technologies, which are included in structure. Research showed that the described approach allows to optimize a set of technologies, ranges of their functioning, switching strategy, and also process of redistribution of all-system resources on a scientific basis, maximizing "metasystem effect" of integration.

\section{REFERENCES}

[1] D.B. Pogorelov, A.M. Pishchukhin About a choice of economic control levers to the enterprises. Successes modern естествознания. No. 11, 2007, pp 101-102
[2] G.J.Klir Architecture of Systems Problem Solving, with D. Elias, Plenum Press, New York,1985, 354 p.

[3] S.V. Mironov, A.M. Pishchukhin Metasystem approach in management. Orenburg, PMC OSU, 2004, 336 p.

[4] A.M. Pishchukhin, A.M. SkamyinMetasystem in product management //Modern high technologies, 2005, No. 11,pp $70-71$.

[5] A.M. Pishchukhin, Y.Y. Grachikov, G.G. Pishchukhina, S.A. Grachikova Metasystem approach to the organization of medical care. Basic researches. No. 1, 2004, p 79

[6] A.M. Pishchukhin, V.V. Tugov, A.V. Tribunskiy Optimum control readiness collecting and preparation system of oil for use.Automation and modern technologies. No 3, 2010, pp $3-5$.

[7] T.I. Korshunova, E.A.Korshunova, T.A. Pishchukhina,A.M. PishchukhinTrajectory strategy of a choice of functioning systems in metasystem.Messenger of Orenburg state university, No. 1, 2003, pp 141-143.

[8] A.M. Pishchukhin, A.V.ShalkinMatrix control systems. Messenger of Orenburg state university, No. 1, 2001, pp 140-145.

[9] A.M. Pishchukhin, E.A.Korshunova Modeling of organizational structure of the enterprise at various stages of life cycle Messenger of Orenburg state university, No. 4, 2009, pp88-93.

[10] A.M. Pishchukhin, A.M. SkamyinAdaptive management of versatile production. Successes of modern natural sciences.No. 10, 2007, p 103. 